\title{
Effect on the Formation of Magnetite Reduced Graphene Oxide with Controlled Stirring Duration
}

\author{
Xin Hui Yau ${ }^{1,2}$, Cheng Seong Khe ${ }^{1,2}$, Mohamed Shuaib Mohamed Saheed ${ }^{1,2}$ and Chin Wei Lai ${ }^{3}$ \\ ${ }^{1}$ Department of Fundamental and Applied Sciences, Universiti Teknologi PETRONAS, Seri Iskandar, 32610 Perak, Malaysia. \\ ${ }^{2}$ Centre of Innovative Nanostructures and Nanodevices (COINN), Universiti Teknologi PETRONAS, 32610 Bandar Seri Iskandar, Perak \\ Darul Ridzuan, Malaysia. \\ ${ }^{3}$ Nanotechnology \& Catalysis Research Centre (NANOCAT), Institute of Postgraduate Studies (IPS), University of Malaya, 3rd Floor, \\ Block A, 50603 Kuala Lumpur, Malaysia.
}

\begin{abstract}
Graphene is a promising material due to its fascinating properties, such as mechanical, electronic and thermal properties. Graphene based hybrids materials also have been widely studied due to its wide applications, such as sensors, energy storage and conversion, electronic device and others. The current study presents the synthesis of magnetite-reduced graphene oxide (M-rGO) nanocomposites through in situ chemical synthesis at different stirring durations. This synthesis process involves the redox reaction between the iron(II) salts and graphene oxide (GO) sheets. Various techniques were employed to characterize the synthesized M-rGO nanocomposites. From X-ray diffraction (XRD) results, the crystal structure of M-rGO was found to be independent on the stirring duration. Three magnetite vibrations, D band and G band were observed in Raman spectrum of M-rGO with 24 hours stirring duration. From Fourier transform infrared (FTIR) analysis, M-rGO with 24 hours stirring duration showed the strong intensity of $\mathrm{Fe}-\mathrm{O}$ vibration. Thus, this indicated that a large amount of magnetite nanoparticles were covered on the surface of rGO sheets. This result is further supported by the morphology of nanocomposites from scanning electron microscopy (SEM) and the elemental analysis (EDX). A monolayer of rGO sheet (C=33.79 atomic \%) with full coverage of magnetite nanoparticles $(\mathrm{Fe}=30.20$ atomic \%) was found for the $\mathrm{M}-\mathrm{rGO}$ with 24 hours stirring duration. Overall, M-rGO require 24 hours of continuous stirring to ensure full coverage of magnetite nanoparticles on the surface of rGO sheets.
\end{abstract}

\section{Introduction}

In 2004, Novoselov and Geim were firstly isolated graphene from graphite through a simple mechanical exfoliation method [1]. Their works had opened a series of opportunities in the realization the usage of graphene in various science and technology due to the remarkable physiochemical properties [2]. Graphene is a one atom thick 2D structure with excellent mechanical, electronic and thermal properties [3, 4]. Graphene oxide (GO) is a precursor material for graphene. GO is recognized as the oxidation product of graphite and it contains carboxyl, carbonyl, epoxyl and hydroxyl groups attached on the graphene sheet and thus can readily disperse in water [5]. Chemical reduction of $\mathrm{GO}, \mathrm{rGO}$, involves the removing of attached oxygen rich functional groups from graphene sheet and is an economical route for the production of graphene in large quantities [6]. Owing to the special surface properties, large surface area and layered structured, GO represents an excellent platform for the developing of graphene-based hybrids materials [7]. Recently, these graphene based hybrids materials have been intensively studied due to their wide applications $[8,9]$.

Iron oxide nanoparticles have also been widely used for incorporation into graphene based materials [10-12]. Among them, magnetite $\left(\mathrm{Fe}_{3} \mathrm{O}_{4}\right)$ nanoparticles are the most selective materials for the combination with graphene/graphene oxide based materials [11, 13, 14]. Magnetite is also called iron (II,III) oxide as the formula of magnetite, $\mathrm{Fe}_{3} \mathrm{O}_{4}$, can be shown as $\left(\mathrm{FeO} \cdot \mathrm{Fe}_{2} \mathrm{O}_{3}\right)$. Magnetite differ from other iron oxides as it contains both $\mathrm{Fe}^{2+}$ and $\mathrm{Fe}^{3+}$ ions, and these enables magnetite to possess various fascinating properties including superparamagnetic, high coercivity, low curie temperature and high magnetic susceptibility $[15,16]$. Therefore, the combination of magnetite nanoparticles with graphene would provide a new and functional hybrid with synergistic interaction between the components.

There are several methods used for the preparation of magnetite graphene based nanocomposites [17-19]. Basically, these methods are classified into two groups, one of which is decorating of $\mathrm{Fe}_{3} \mathrm{O}_{4}$ on $\mathrm{rGO}$ sheet and another one is through chemical reaction of $\mathrm{GO}$ with ferrous chloride or ferrous sulphate to form magnetite 
reduced graphene oxide (M-rGO) nanocomposites. The easier method is the in situ chemical synthesis of M-rGO nanocomposites [20]. The formation of M-rGO nanocomposites involves redox reaction in which the oxidation state of iron (II) is increased to iron (III) while $\mathrm{GO}$ is reduced to $\mathrm{rGO}$.

In the present study, we report one of the most convenient methods employed for the synthesis of M-rGO nanocomposites by using in situ chemical synthesis. This study aims to investigate the effect of stirring duration on formation of M-rGO nanocomposites. Hence, the stirring duration from 0.5 hours to 24 hours were examined. The characteristic of the obtained samples were studied by X-ray diffraction (XRD), Fourier transform infrared spectroscopy (FTIR), Raman spectroscopy and field emission scanning electron microscope (FESEM) equipped with energy dispersive $\mathrm{X}$-ray (EDX) detector.

\section{Materials and methods}

\subsection{Materials}

Graphite flakes was purchased from Sigma Aldrich. Sulfuric acid $\left(\mathrm{H}_{2} \mathrm{SO}_{4}, 98 \%\right)$, phosphoric acid $\left(\mathrm{H}_{3} \mathrm{PO}_{4}\right.$, $85 \%$ ), potassium permanganate $\left(\mathrm{KMnO}_{4}, 99.9 \%\right)$, hydrogen peroxide $\left(\mathrm{H}_{2} \mathrm{O}_{2}, 30 \%\right)$, hydrochloric acid $(\mathrm{HCl}$, $37 \%$ ), ammonium hydroxide $\left(\mathrm{NH}_{4} \mathrm{OH}, 25 \%\right)$ and iron(II) sulphate heptahydrate $\left(\mathrm{FeSO}_{4} .7 \mathrm{H}_{2} \mathrm{O}, 99.5 \%\right)$ were purchased from Merck. All chemicals were of analytical grade and used without any purification. Deionized water was used in all experiments.

\subsection{Synthesis of magnetite reduced graphene oxide (M-rGO)}

Graphene oxide (GO) in this work was prepared from oxidizing the graphite flakes following the method proposed by Marcano and his co-workers [21]. In a typical method, a 9:1 mixture of $\mathrm{H}_{2} \mathrm{SO}_{4}-\mathrm{H}_{3} \mathrm{PO}_{4}$ $(180: 20 \mathrm{~mL})$ was added to a mixture of graphite flakes $(1.5 \mathrm{~g})$ and $\mathrm{KMnO}_{4}(9.0 \mathrm{~g})$. The mixture was heated to $50{ }^{\circ} \mathrm{C}$ and left stirred for three days to ensure complete oxidation of graphite. $\mathrm{H}_{2} \mathrm{O}_{2}$ was then added to stop the oxidation reaction. The $\mathrm{GO}$ was obtained and washed three times with $\mathrm{HCl}$ and deionized water through centrifugation until $\mathrm{pH} 4-5$ was achieved. The settlements (GO) obtained were then dried in $40{ }^{\circ} \mathrm{C}$ oven to form $\mathrm{GO}$ solid. Magnetite reduced graphene oxide (M-rGO) was prepared by in situ chemical method. GO (0.1g) was dispersed in the deionized water with the aid of sonication for 20 mins. $\mathrm{NH}_{4} \mathrm{OH}(10 \mathrm{~mL})$ was then added to the GO solution until the $\mathrm{pH} 11-12$ was obtained. Subsequently, $\mathrm{FeSO}_{4}(0.036 \mathrm{M})$ was added slowly to the GO solution under the magnetic stirring and stirred at different stirring duration $(0.5,3,12,24 \mathrm{~h})$ at room temperature. Black solution was obtained once stirring duration was completed. The solution was then centrifuged and washed three times with deionized water at $4000 \mathrm{rpm}$ for 10 mins. The M-rGO obtained was then dried in $30{ }^{\circ} \mathrm{C}$ oven for overnight. The parameter and designations of the prepared samples were shown in Table 1.

Table 1. Synthesis parameter and designations of M-rGO.

\begin{tabular}{|c|c|}
\hline Sample & $\begin{array}{c}\text { Stirring Duration } \\
\text { (hours, } \mathrm{h} \text { ) }\end{array}$ \\
\hline M-rGO1 & 0.5 \\
\hline M-rGO2 & 3 \\
\hline M-rGO3 & 12 \\
\hline M-rGO4 & 24 \\
\hline
\end{tabular}

\subsection{Instrumentations}

The M-rGO nanocomposites obtained were stored for characterization. Bruker D8 advance diffractometer (XRD) with $\mathrm{CuK} \alpha$ radiation was used for identify the crystalline structure of $\mathrm{M}-\mathrm{rGO}$ samples. The M-rGO pellets were prepared using $\mathrm{KBr}$ and the samples were scanned in the range of $500-4000 \mathrm{~cm}^{-1}$ to obtain the FTIR spectra by using a Perkin Elmer Spectrum BX Fourier transform infrared spectroscopy (FTIR). Raman spectrum is performed using Horiba Jobin-Yvon HR800 Raman Spectroscopy equipped with a $514.5 \mathrm{~nm}$ laser source to examine the change in chemical and crystalline structure during the synthesis process. Surface morphology and the elemental analysis of M-rGO nanocomposites were studied using ZEISS Leo Supra 55 variable pressure field emission scanning electron microscope (FESEM) equipped with energy dispersive X-ray (EDX) detector.

\section{Results and discussion}

In this experiment, the influence of stirring duration on crystalline nature of the samples was investigated through X-ray diffraction (XRD) analysis. Figure 1(a-d) shows the XRD patterns of M-rGO nanocomposites synthesized at different stirring durations $(0.5,3,12$ and $24 \mathrm{~h})$. In general, M-rGO in Figure 1(a-d) consist of six diffraction peaks which located at $2 \theta=30.0^{\circ}, 35.4^{\circ}, 43.0^{\circ}, 53.3^{\circ}$, $57.0^{\circ}$ and $62.5^{\circ}$, corresponding to the (220), (311), (400), (422), (511) and (440) plane of reflections of magnetite $\left(\mathrm{Fe}_{3} \mathrm{O}_{4}\right)$ (JCPDS no. 19-0629). According to Low et al., $\mathrm{XRD}$ spectrum of $\mathrm{GO}$ has a diffraction peak at $2 \theta=8.59^{\circ}$ [22]. The disappearance of this peak in the XRD spectra of $\mathrm{M}-\mathrm{rGO}$ indicated that the oxygen functional groups were removed [22] and rGO was formed. The disappearance of GO reflection peak and the appearance magnetite reflection peaks in the XRD spectra have confirmed that the M-rGO nanocomposites were successfully synthesized through in situ chemical synthesis. The XRD results clearly show that the crystal 
structure of $\mathrm{M}-\mathrm{rGO}$ is independent on the stirring duration.

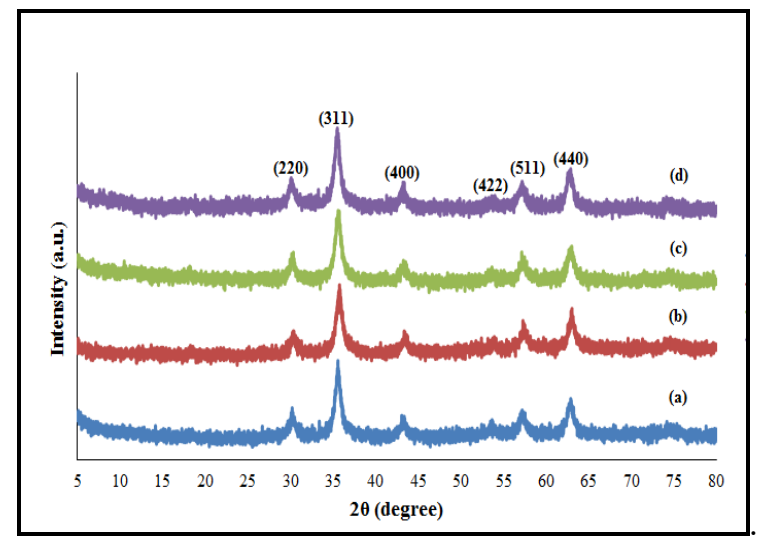

Figure 1. XRD patterns of (a) M-rGO1, (b) M-rGO2, (c) M-rGO3 and (d) M-rGO4.

Fourier transform infrared spectroscopy (FTIR) analysis was carried out to investigate the changes of functional groups of M-rGO nanocomposites that synthesized using different stirring duration. Figure 2(a-d) exhibits the FTIR spectra of various M-rGO nanocomposites. Generally, the FTIR spectra of M-rGO in Figure 2(a-d) have the same characteristic peaks, such as the hydroxyl broad peak $(-\mathrm{OH})$ at about $3420 \mathrm{~cm}^{-1}$, $\mathrm{C}=\mathrm{C}$ stretching peak at $1623 \mathrm{~cm}^{-1}$, epoxy stretching peak (C-O-C) at $1050 \mathrm{~cm}^{-1}$, and Fe-O lattice vibration at $570 \mathrm{~cm}^{-1}[23,24]$. However, it can be noticed that the high intensity peak for Fe-O was present for the sample M-rGO4 (Figure 2d). In other words, the M-rGO synthesized at 24 hours continuously stirring has the strongest magnetite $\mathrm{Fe}-\mathrm{O}$ lattice vibration.

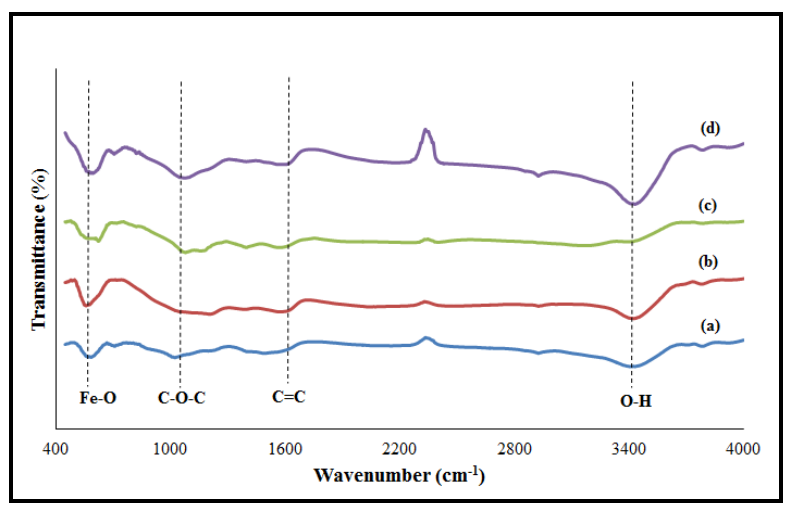

Figure 2. FTIR spectra of (a) M-rGO1, (b) M-rGO2, (c) M-rGO3 and (d) M-rGO4.

Raman spectroscopy is an important tool for the characterization of graphene based nanocomposites. Figure 3 compare the Raman spectra of M-rGO nanocomposites synthesized using various stirring durations. In the M-rGO materials, there are total of five vibrations, including two fundamental vibrations, namely the $D$ and $G$ vibration band, which located at 1350 and $1580 \mathrm{~cm}^{-1}$, respectively, and other vibration bands at 212 , 271 and $385 \mathrm{~cm}^{-1}$ are originated from magnetite (M). D band is the disorder mode due to the oxidation of graphite, whereas the $\mathrm{G}$ band is the first order $\mathrm{E}_{2} \mathrm{~g}$ mode from $\mathrm{sp}^{2}$ carbon domain [25]. The Raman spectrum of M-rGO1 as shown in Figure 3(a) has only a D band and a $\mathrm{G}$ band. This indicated that a small amount of magnetite nanoparticles are attached on the surface of rGO sheet and thus unable to detect the magnetite vibration in Raman analysis. By contrast, when the M-rGO stirring duration increased from $0.5 \mathrm{~h}$ to $24 \mathrm{~h}$, the Raman spectrum of M-rGO2 (Figure 3b), M-rGO3 (Figure 3c) and M-rGO4 (Figure 3d), all displayed five vibration peaks in Raman spectra.

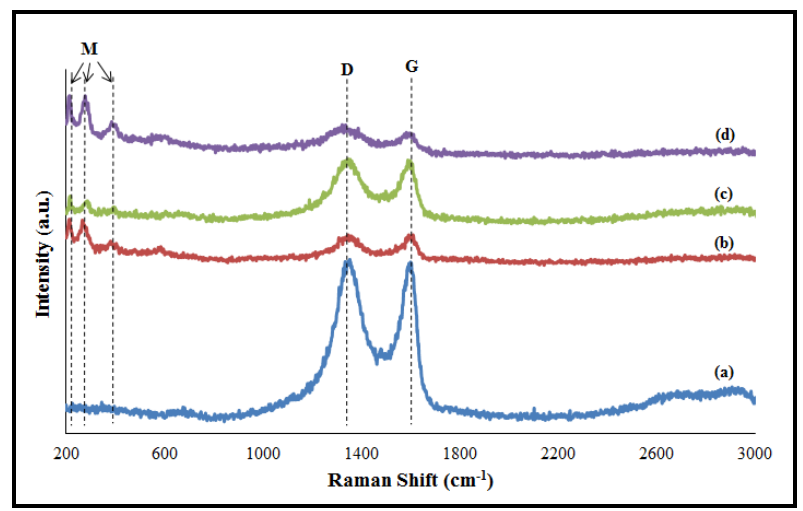

Figure 3. Raman spectra of (a) M-rGO1, (b) M-rGO2, (c) M-rGO3 and (d) M-rGO4.

Field emission scanning electron microscopy (FESEM) was carried out in order to investigate the morphology of the synthesized M-rGO samples. Figure 4(a-d) shows the FESEM images of M-rGO samples after various stirring durations $(0.5,3,12$ and $24 \mathrm{~h})$. FESEM images in Figure 4(a) shows that a layered of rGO sheet with little amount of magnetite nanoparticles attached on it. This result again proved that 0.5 hours stirring duration is not enough for the formation of M-rGO nanocomposites. When the stirring duration increased to 3 hours (Figure 4b) and 12 hours (Figure 4c), more magnetite nanoparticles were attached on $\mathrm{rGO}$ sheet. Meanwhile, M-rGO with 24 hours continuously stirring as shown in figure 4(d) has a fully coverage of magnetite nanoparticles on the monolayer of rGO sheet. The FESEM results clarify that as the stirring duration increase, more magnetite nanoparticles are attached on the rGO sheet.
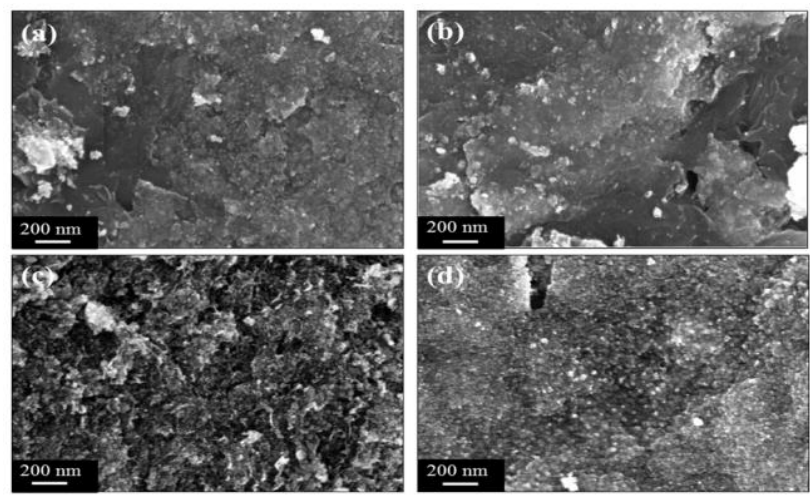

Figure 4. FESEM images of (a) M-rGO1, (b) M-rGO2, (c) M-rGO3 and (d) M-rGO4. 
Table 2 indicated the composition of synthesized M-rGO samples obtained from the energy dispersive X-ray (EDX) detector from FESEM instrument. From EDX results, the M-rGO4 has the lowest amount of carbon, C with 33.79 atomic \% and highest amount of iron, Fe with 30.20 atomic \%. The ratio of iron to carbon $(\mathrm{Fe} / \mathrm{C})$ was calculated and M-rGO4 achieved the highest $\mathrm{Fe} / \mathrm{C}$ ratio $(\mathrm{Fe} / \mathrm{C}=0.89)$. Undeniably, $\mathrm{M}-\mathrm{rGO}$ with 24 hours continuously stirring has the higher amount of magnetite nanoparticles attached on the rGO sheet and the lowest amount of carbon detector suggested that the magnetite nanoparticles were well covered on the surface of rGO sheet.

Table 2. Summarize of EDX analysis (in atomic \%) of M-rGO.

\begin{tabular}{|c|c|c|c|c|}
\hline Sample & $\mathrm{C}(\%)$ & $\mathrm{O}(\%)$ & $\mathrm{Fe}(\%)$ & Fe/C ratio \\
\hline M-rGO1 & 37.02 & 46.81 & 16.17 & 0.44 \\
\hline M-rGO2 & 38.35 & 45.19 & 16.46 & 0.43 \\
\hline M-rGO3 & 45.82 & 36.29 & 17.89 & 0.39 \\
\hline M-rGO4 & 33.79 & 36.01 & 30.20 & 0.89 \\
\hline
\end{tabular}

Based on the results obtained, a brief and plausible interaction mechanism between the iron(II) salt and GO in aqueous was proposed to explain the formation of M-rGO nanocomposites. Both iron(II) salt and GO in aqueous solution interact through coordinate (dative covalent) bonding. Iron(II) salt in aqueous solution tend to act as cations, which are $\mathrm{Fe}^{2+}$. Cations are electron deficient with unoccupied orbitals. Meanwhile, the GO surface consist a lot of electron rich oxygen atoms, such as hydroxyl (-OH) and epoxy (-O-) groups with lone pairs of electrons. The coordinate bond is formed between the $\mathrm{Fe}^{2+}$ and oxygen atoms of $\mathrm{GO}$, thus forming the M-rGO nanocomposites.

\section{Conclusion}

In conclusion, the magnetite reduced graphene oxide (M-rGO) nanocomposites were successfully synthesized through in situ chemical synthesis. The longer the stirring duration, more magnetite nanoparticles were attached on the surface of $\mathrm{rGO}$ sheet. M-rGO4 or M-rGO with 24 hours of continuously stirring is the best sample among others as it contain low amount of carbon atom (33.79 atomic\%) and high amount of iron atom (30.20 atomic\%). A monolayer of rGO sheet with the fully coverage of magnetite nanoparticles was obtained for the M-rGO samples with 24 hours stirring duration.

\section{Acknowledgments}

Authors acknowledge Yayasan Universiti Teknologi PETRONAS (YUTP) Grant (YUTP 0153AA-E40) and
Graduate Assistantship program from the Universiti Teknologi PETRONAS for providing financial assistance.

\section{References}

1. K. S. Novoselov, A. K. Geim, S. V. Morozov, D. Jiang, Y. Zhang, S. V. Dubonos, I. V. Grigorieva and A. A. Firsov, Science 306 (5696), 666-669 (2004).

2. K. S. Novoselov, V. Fal, L. Colombo, P. Gellert, M. Schwab and K. Kim, Nature 490 (7419), 192 (2012).

3. A. K. Geim and K. S. Novoselov, Nat. Mater. 6 (3), 183 (2007).

4. X. Huang, Z. Yin, S. Wu, X. Qi, Q. He, Q. Zhang, Q. Yan, F. Boey and H. Zhang, Small 7 (14), 18761902 (2011).

5. D. Konios, M. M. Stylianakis, E. Stratakis and E. Kymakis, J. Colloid Interface Sci. 430, 108-112 (2014).

6. D. Li, M. B. Müller, S. Gilje, R. B. Kaner and G. G. Wallace, Nat. Nanotechnol. 3 (2), 101 (2008).

7. M. Şinoforoğlu, B. Gür, M. Arık, Y. Onganer and K. Meral, RSC Adv. 3 (29), 11832-11838 (2013).

8. S. Stankovich, D. A. Dikin, G. H. Dommett, K. M. Kohlhaas, E. J. Zimney, E. A. Stach, R. D. Piner, S. T. Nguyen and R. S. Ruoff, Nature 442 (7100), 282 (2006).

9. M. Pumera and C. H. A. Wong, Chem. Soc. Rev. 42 (14), 5987-5995 (2013).

10. B. Saiphaneendra, T. Saxena, S. A. Singh, G. Madras and C. Srivastava, J. Environ. Chem. Eng. 5 (1), 26-37 (2017).

11. R. Hatel, M. Goumri, B. Ratier and M. Baitoul, Mater. Chem. Phys. 193, 156-163 (2017).

12. J. Liu, H. Cao, J. Xiong and Z. Cheng, Cryst. Eng. Comm. 14 (16), 5140-5144 (2012).

13. M. Liu, C. Chen, J. Hu, X. Wu and X. Wang, The J. Phys. Chem. C 115 (51), 25234-25240 (2011).

14. V. Chandra, J. Park, Y. Chun, J. W. Lee, I.-C. Hwang and K. S. Kim, ACS Nano 4 (7), 3979-3986 (2010).

15. W. Wu, Q. He and C. Jiang, Nanoscale Res. Lett. 3 (11), 397 (2008).

16. L. Blaney, Lehigh Rev. 15, 33-81(2007).

17. V. Hoan, N. Thi, A. Thu, N. Thi, H. V. Duc, N. D. Cuong, D. Quang Khieu and V. Vo, J. Chem. 2016 (2016).

18. Y. Sun, W. Zhang, H. Yu, C. Hou, D.-s. Li, Y. Zhang and Y. Liu, J. Alloys Compd. 638, 182-187 (2015).

19. A. Meidanchi and O. Akhavan, Carbon 69, 230-238 (2014).

20. P. S. Teo, H. N. Lim, N. M. Huang, C. H. Chia and I. Harrison, Ceram. Int. 38 (8), 6411-6416 (2012).

21. D. C. Marcano, D. V. Kosynkin, J. M. Berlin, A. Sinitskii, Z. Sun, A. Slesarev, L. B. Alemany, W. Lu and J. M. Tour, ACS Nano 4 (8), 4806-4814 (2010).

22. F. W. Low, C. W. Lai and S. B. A. Hamid, Ceram Int. 41 (4), 5798-5806 (2015).

23. J. Liang, H. Li, J. Yan and W. Hou, Energy \& Fuels 28 (9), 6172-6178 (2014). 
24. H. Wang, J. Liu, H. Xu, Z. Ma, W. Jia and S. Ren, RSC Advances 6 (108), 106297-106307 (2016).
25. K. N. Kudin, B. Ozbas, H. C. Schniepp, R. K. Prud'Homme, I. A. Aksay and R. Car, Nano Lett. 8 (1), 36-41 (2008). 